Note

\section{Nifedipine Augments Haloperidol in the Treatment of Tourette Syndrome}

Norman E. Alessi, MD, Mark E. Walden, MD, and Paul S. Hsieh

Pediatr Neurol 1988;4:191.

In anecdotal case reports, verapamil and nifedipine, calcium channel blockers, have provided rapid and dramatic relief in refractory Tourette syndrome [1,2]. The following report illustrates the use of nifedipine to augment haloperidol in the treatment of a child who did not respond when either drug was used alone.

This 9-year-old boy was referred for intractable Tourette syndrome over a 3 year period with associated attention deficit hyperactivity disorder. His tics had worsened over the previous 5 months, making fine motor control impossible. He had received a variety of medications, including clonidine, haloperidol, pimozide, and fluphenazine, all of which proved ineffective. Neurologic examination, including cranial computed tomography, electroencephalography, and magnetic resonance imaging, was normal.

The patient received a therapeutic trial of nifedipine; tics and vital signs were monitored. After 1 week of therapy there was neither relief of symptoms, nor presence of side effects. Blood pressure and pulse remained stable. With the addition of haloperidol ( $2 \mathrm{mg} /$ day orally in 2 divided doses), there was a significant reduction in tics and a marked improvement in attention and school performance.

Nifedipine was discontinued to determine whether the observed improvement was due to haloperidol. Within 2 days the tics worsened and his attention deficit returned to pretreatment levels. To determine whether the deterioration in function was due to a placebo effect, a placebo was administered ( 3 tablets per day at equal intervals); the patient and patient's family were told that it was a "medication similar to nifedipine." The patient continued to receive the placebo for 10 days, during which time his tics and attentional difficulties continued. After 10 days, nifedipine therapy was reinstated. Within 2 weeks the patient's tics were markedly improved. His school performance also improved because of an increase in attention, a decrease in impulsivity, a decrease in the frequency and severity of tics, and an improvement in fine motor control.

This is the first report to systematically demonstrate the use of nifedipine to augment haloperidol treatment of Tourette syndrome. These results suggest that an alternative in the treatment of Tourette syndrome in treatmentresistant cases is the combination of haloperidol and nifedipine. In addition to the marked reduction in tics, characteristics of attention deficit disorder were affected: attention improved and impulsivity decreased. The precise basis of the psychopharmacologic impact remains unclear; however, neither nifedipine nor haloperidol alone altered his course. We postulate that the effect observed in this patient represents functional reduction in dopamine receptors with the chronic administration of nifedipine, with a resulting increased efficacy of haloperidol [3]. Further studies are indicated to substantiate this effect, and if present, to determine its mechanism of action.

\section{References}

[1] Goldstein JA. Nifedipine treatment of Tourette's syndrome. J Clin Psychiatry 1984;45:360.

[2] Walsh TL, Lavenstein B, Licamele WL, et al. Calcium antagonists in the treatment of Tourette's disorder Am J Psychiatry 1986; 143:1467-8.

[3] Tecott LH, Kwant LL, Uhr S, et al. Differential modulation of dopamine D2 receptors by chronic haloperidol, nifedipine, and pimozıde. Biol Psychiatry 1986;21:1114-22.

From the Department of Psychiatry; Universıty of Mıchigan; Ann Arbor, Michigan.

Communications should be addressed to: Dr. Alessi: Department of Psychiatry: University of Michigan Medical Center 1500 East Medical Center Drive; Ann Arbor, Michigan 48109-0706. Received February 17, 1988; accepted March 31, 1988. 